\title{
SUB-PHYLUM CRANIATA (VERTEBRATA)
}

\section{INTRODUCTION}

$\mathrm{V}$ ARIOUS classifications of Vertebrata have been proposed, but agreement has been reached on only one point-i.e. that this great group is essentially divisible into two unequal sections: (I) The jawless Agnatha (composed of a small number of primitive but highly specialised fish-like animals), and (2) the Gnathostomata, which embraces all other vertebrates from the true fishes to the Mammalia, including Man. In the past each of these distinctive assemblages has been sometimes accorded the rank of sub-phylum, but, however radically they differ in their buccal and visceral skeletal anatomy in the adult, such an arrangement seems untenable when we consider the criteria on which the three more primitive chordate sub-phyla have been erected and, equally, when we survey the many fundamental similarities existing between these two craniate groups. In recent years there has been a tendency to consider the Agnatha and Gnathostomata as 'branches ', 'groups ', or 'super-classes ' of a single, and final, sub-phylum of the animal kingdom, and that is the arrangement followed here.

We are faced now, as we shall be confronted again, with the perennially vexed question as to what classificatory status various included groups should be accorded. For example, different authorities assign the Euphanerida (p. I67) variously to Family, Order, and Class. At the present stage of our knowledge, the conventional arrangements of many fossil, and even living, groups are essentially provisional and arbitrary. We shall see later, for example, that the 'Orders' erected by ornithologists have no validity whatever when judged by criteria employed by ichthyologists and herpetologists. No arrangement in any group can hope to be universally agreed at the present time. As mentioned above, several opinions are available concerning the most valid form of representation within each branch of the sub-phylum Vertebrata. ${ }^{1}$

1 Goodrich recognised three branches of craniates: the Ostracodermi, including all the fossil agnathans; the Cyclostomata, including the lampreys and hags; and the Gnathostomata. Stensiö and Jarvik classify the Agnatha into the Sub-classes Cephalaspidomorphi, including the cephalaspids, anaspids, and lampreys; and the Pteraspidomorphi, including the pteraspids and hags. Romer includes all except the pteraspids in the Sub-class Cephalaspidomorphi. 